\title{
Information imparfaite et économie publique
}

Monsieur Jean-Jacques Laffont

\section{Citer ce document / Cite this document :}

Laffont Jean-Jacques. Information imparfaite et économie publique. In: Revue économique, volume 33, n¹, 1982. pp. 5-29; http://www.persee.fr/doc/reco_0035-2764_1982_num_33_1_408641

Document généré le 28/05/2016 


\title{
Résumé
}

Une caractéristique essentielle des systèmes économiques est la décentralisation de l'information. Lorsqu'une décision collective doit être prise, le décideur social doit rassembler une information dont chaque agent possède des éléments qu'il est le seul à connaître. Par suite il se pose le problème du comportement straté-gique vis-à-vis de cette information détenue de façon privative. Etant donné une fonction de choix social qui associe aux caractéristiques informationnelles des agents une décision collective souhaitée par le décideur social, comment peut-on la concrétiser, c'est-à-dire trouver un jeu dont les équilibres stratégiques condui-sent à la décision souhaitée ?

L'objectif de cette note est d'exposer la problématique générale de la concrétisation et d'en présenter quelques résultats pour des problèmes classiques d'économie publique.

\begin{abstract}
Imperfect information and public economics

Jean-Jacques Laffont

Decentralization of information is an essential characteristic of economic Systems. To take a collective decision, the decision maker must gather pieces of information which are privately owned. Strategic behavior with respect to this information must then be expected. Given a social choice function which associates to agents' informational characteristics a collective décision desired by thé décision maker, how is it possible to implement it, i.e. to find a game the strategic equilibria of which lead to the desired decision. The purpose of this note is to present the implementation problem and to give some results obtained in public economics.
\end{abstract}




\section{INFORMATION IMPARFAITE ET ECONOMIE PUBLIQUE *}

$\mathrm{U}^{\mathrm{s}}$

NE caractéristique essentielle des systèmes économiques est la décentralisation de l'information. Lorsqu'une décision collective doit être prise, que ce soit le choix du niveau d'une taxe qui fait internaliser un effet externe, le choix du niveau d'un bien public, le choix du niveau de production d'une entreprise à rendements croissants, ou encore le choix d'ume constitution, le décideur social doit rassembler une information très décentralisée; chaque agent possède des éléments d'information qu'il est le seul à connaître. Par suite, il se pose le problème d'un comportement stratégique vis-à-vis de cette information détenue de façon privative.

Etant donné une fonction de choix social qui associe aux caractéristiques informationnelles des agents une décision collective souhaitée par le décideur social ou Centre, comment peut-on la concrétiser ${ }^{1}$, c'està-dire trouver un jeu dont les équilibres stratégiques conduisent à la décision souhaitée.

L'objectif de cette note est d'exposer la problématique générale de la concrétisation et d'en présenter quelques résultats pour des problèmes classiques d'économie publique.

* Cette note constitue le cahier 8101 de la série GREMAQ, ERA 937 ; elle a pour objectif d'initier à la théorie de l'économie publique en information imparfaite. Plutôt que d'essayer de fournir un tour d'horizon de ce clomaine en plein développement (voir cahier 8102 pour cela), j’ai cherché à pénétrer jusqu'au cœur du sujet en privilégiant un point de -vue particulier.

1. Pour la traduction du verbe to implement, j'ai suivi la suggestion d'un lecteur anonyme de la revue, malgæé la lourdeur des dérivés, concrétisation, concrẻtisable, etc... La traduction par réaliser, réalisation, réalisable conduit à des confusions, car une allocation réalisable est une allocation physiquement ou techniquement possible. 


\section{LA PROBLEMATIQUE \\ DE LA CONCRETISATION}

Considérons une société composée de I agents et soit A l'ensemble des états sociaux réalisables. Les préférences de l'agent $i$ sont représentées par un préordre ${ }^{2} \mathbf{R}^{i}$. Parfois, il sera commode de limiter l'espace des préférences admissibles de l'agent $i$. Soit (A) ${ }^{i}$ un espace de caractéristiques pour l'agent $i$ : à tout $\theta^{i} \in \mathbb{A}^{i}$ est associé un préordre $\mathbf{R}^{i}\left(\theta^{i}\right)$ pour lagent $i$ par une application $\mathrm{R}^{i}($.$) connue de tous.$

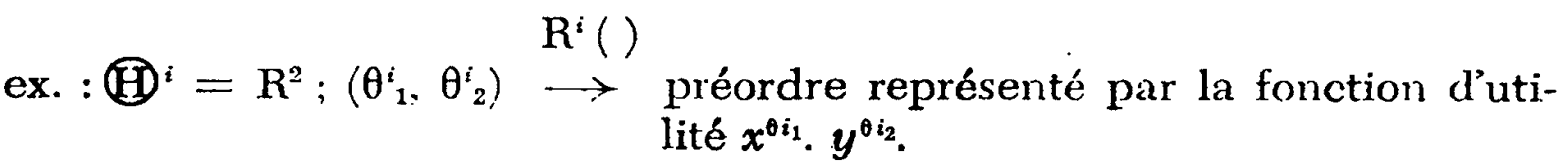

S'il n'existe aucume restriction sur les préordres admissibles, (16) représente l'espace des préordres sur. $A$, et $R^{i}($.$) est l'application$ identité.

La vraie valeur de la caractéristique $\theta^{i}$ est connue seulement de l'agent $i$; l'information est décentralisée. Le Centre doit choisir un état social sur la base de messages que lui transmettent les agents sur leurs caractéristiques privées. Etant donné que chaque agent cherche à utiliser à son avantage l'ignorance par le Centre de ses propres caractéristiques, la théorie des incitations étudie ce que peut néanmoins réaliser le Centre à l’aide de mécanismes destinés à surmonter au moindre coût son «gap » informationnel.

Les définitions suivantes vont nous permettre de préciser cette problématique.

Une fonction de choix social, $f$, est une application qui associe à tout vecteur de caractéristiques,

$\theta \equiv\left(\theta^{1}, \ldots, \theta^{\mathrm{r}}\right) \in \mathbb{A} \equiv \prod_{i=1}^{\mathbf{I}} \mathbb{A}^{i}$, un état social réalisable $f(\theta) \in \mathbf{A}$.

Un mécanisme est un I-uple d'espaces de messages $\mathrm{M} \equiv\left(\mathrm{M}^{1}, \ldots, \mathrm{M}^{\mathrm{r}}\right)$ et une fonction $g(\cdot)$ qui associe à tout I-uple de messages $m \equiv\left(m^{1}, \ldots, m^{\mathrm{I}}\right)$ un état social réalisable $g(m) \in \mathrm{A}$.

L'agent $i$ doit annoncer un message $m^{i}$, sachant que le Centre choisit l'état social à l'aide du mécanisme. Un mécanisme définit donc un jeu à information incomplète pour lequel il faut choisir un concept d'équilibre, noté $c$.

2. Nous appelons ici préordre une relation binaire totale, réflexive et transitive. 
Soit $\mathrm{E}_{g . c}(\cdot)$ lapplication (qui associe à tout I-uple cle caractéristiques vraies $\theta$, les messages déquilibre de ce jeu pour le concept d'équilibre $c$.

Un mécanisme (M, g) concrétiss me fonction de choix social $f($. pour le concept d'éculilibre c si :

$$
\forall \theta \in \mathbb{A} \quad \because\left(\mathrm{F}_{, \ldots,}(\theta)\right) \equiv f(\theta)
$$

La notion de concrétisation peut ètre illustrée par le schéma suivant

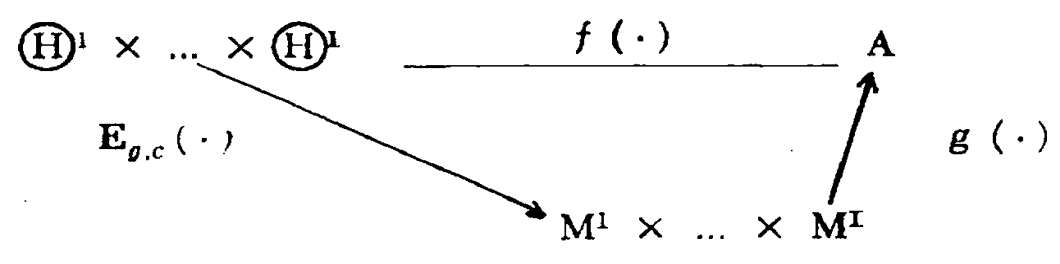

Deux exemples de concepts d'équilibre vont permettre de rendre plus concret ce schéma.

了

Equilibre dominant : $c \equiv d$

Un I-uple de messages $\left(m^{* 1}, \ldots, m^{* 1}\right) \in M$ est un équilibre dominant si et seulement si :

$$
\begin{aligned}
\forall i=1, \ldots, \mathrm{I} \quad \mathrm{g}\left(m^{* i}, m^{-i}\right) & \mathbb{R}^{i}\left(\theta^{i}\right) g\left(m^{i}, m^{-i}\right) \\
& \forall m^{i} \in \mathrm{M}^{i} \\
& \forall m^{-i} \in \prod_{j \neq i} \mathrm{M}^{j}
\end{aligned}
$$

où

$$
\begin{aligned}
m^{-i} & \equiv\left(m^{1}, ., m^{i-1}, m^{i+1}, \ldots, m^{\mathrm{I}}\right) \\
\left(m^{i}, m^{-i}\right) & \equiv\left(m^{1}, \ldots, m^{i-1}, m^{i}, m^{i+1}, \ldots, m^{\mathrm{I}}\right)
\end{aligned}
$$

Quels que soient les messages des autres agents, le choix du message $m^{* i}$ par l'agent $i$ conduit à un état social qui est préféré ou indifférent à tout autre état social qui aurait pu être obtenu par un autre message.

A tout $\theta \in \mathbb{H}, \mathrm{E}_{g . d}($.$) associe l'ensemble des équilibres dominants.$ La fonction $g($.$) traduit ces messages d'équilibre en états sociaux.$ 
Equilibre de Nash : $c \equiv n$

Un I-uple de messages $\left(m^{* 1}, \ldots, m^{* I}\right)$ est un équilibre de Nash si et seulement si :

$$
\begin{array}{r}
\forall i=1, \ldots, \mathrm{I} \quad g\left(m^{* i}, m^{*-i}\right) \mathrm{R}^{i}\left(\theta^{i}\right) g\left(m^{i}, m^{*-i}\right) \\
\forall m^{i} \in \mathrm{M}^{i}
\end{array}
$$

Etant donné les messages des autres agents, $m^{*-i}$, le choix du message $m^{* i}$ est au moins aussi bon que tout autre message qu'il pourrait choisir. A tout $\theta \in \mathbb{(}), E_{g . n}(\cdot)$ associe l'ensemble des I-uples des messages, équilibres de Nash.

C'est le concept d'équilibre choisi qui détermine la force incitative de la concrétisation obtenue. Ainsi la concrétisation en équilibres dominants apparaît-elle comme la plus satisfaisante au sens suivant : si les agents ont vraiment des messages dominants, on peut s'attendre de façon très légitime à ce qu'ils les utilisent. Les agents ont de très fortes incitations à adopter le comportement que l'on attend d'eux. Par contre la concrétisation en équilibres de Nash apparaît beaucoup plus faible : est-on sûr que les agents parviendront à un équilibre de Nash? Adopteront-ils le comportement de Nash qui est assez naif ?

Lorsque le concept d'équilibre utilisé est l'équilibre en messages dominants, la théorie des incitations est grandement simplifiée par le principe de révélation.

Nous dirons qu'un mécanisme est direct si l'espace des messages de l'agent $i, \mathrm{M}^{i}$, coïncide avec l'espace de ses caractéristiques (H) $^{i}, i=1, \ldots, \mathrm{I}$.

Un mécanisme direct est dit révélateur si $\mathrm{E}_{g, c}(\theta)=\cdot \theta$, c'est-à-dire si les messages d'équilibre coïncident avec l'annonce des vraies caractéristiques.

D’après le schéma ci-dessus, il est clair que, lorsqu'un mécanisme est révélateur, $g(\theta) \equiv f(\theta)$. Nous pouvons maintenant énoncer :

Théorème 1. Le principe de révélation (Gibbard [1973])

Soit ( $g, M$ ) un mécanisme qui concrétise la fonction de choix social $f($.$) pour le concept d'équilibre dominant. Alors, il existe un$ mécanisme direct révélateur $(\Psi,(H)$ qui concrétise $f($.$) en équilibres$ dominants. 
Démonstration. Soit $m^{* 1}\left(\theta^{1}\right), \ldots, m^{* 1}\left(\theta^{\mathrm{I}}\right)$ un I-uple de messages dominants pour $(g, M)$. Définissons $\Psi(\cdot)$ par :

$$
\Psi(\theta) \equiv g\left(\mathrm{E}_{g, d}(\theta)\right) .
$$

Par définition, $\Psi(\theta) \equiv f(\theta)$; ( $\Psi$, (iD) est bien un mécanisme direct. Supposons que l'annonce de sa raie caractéristique ne soit pas un message dominant pour l'agent $i$. Il existe alors $\left(\hat{\theta}^{i}, \theta^{-i}\right) \in \mathbb{B}$, tel que :

$$
\Psi\left(\tilde{\theta}^{i}, \theta^{-i}\right) P^{i}\left(\theta^{i}\right) \Psi\left(\theta^{i}, \theta^{-i}\right)
$$

où $\mathrm{P}^{i}\left(\theta^{i}\right)$ est la relation de préférence stricte associée à $\mathrm{R}^{i}\left(\theta^{i}\right)$.

Par définition, (1) peut se réécrire :

$$
g\left(\mathrm{E}_{o, d}\left(\tilde{\theta}^{i}, \theta^{-i}\right)\right) \quad \mathrm{P}^{i}\left(\theta^{i}\right) \quad g\left(\mathrm{E}_{o, d}\left(\theta^{i}, \theta^{-i}\right)\right)
$$

soit

$$
\left(m^{* 1}, \ldots, m^{* 1}, \ldots, \tilde{m}^{* I}\right) \in E_{g, d}\left(\tilde{\theta}^{i}, \theta^{-i}\right) .
$$

(2) nous dit qu'il existe :

$$
\left(m^{* 1}, \ldots, m^{*(i-1)}, \tilde{m}^{i}, m^{*(i+1)}, \ldots, m^{* 1}\right) \in \mathbf{E}_{g, d}\left(\tilde{\theta}^{i}, \theta^{-i}\right)
$$

tel que

$$
\begin{aligned}
g\left(m^{* 1}, \ldots, m^{*(i-1)}, \dot{m}^{i}, m^{*(i+1)}, \ldots, m^{* 1}\right) & P^{i}\left(\theta^{i}\right) \\
& \quad g\left(m^{* 1}, \ldots, m^{* i}, \ldots, m^{* 1}\right)
\end{aligned}
$$

ce qui contredit l'hypothèse

$$
\left(m^{* 1}, \ldots, m^{* i}, \ldots, m^{* 1}\right) \in \mathrm{E}_{g, d}\left(\theta^{i}, \theta^{-i}\right) \quad \text { Q.E.D. }
$$

Ainsi, toutes les fonctions de choix social qui peuvent être concrétisées par les équilibres dominants de mécanismes complexes peuvent l'être par des mécanismes directs révélateurs. Il suffit donc de restreindre l'étude aux mécanismes directs révélateurs lorsqu'on étudie la concrétisation en équilibres dominants.

La deuxième idée générale que je souhaite souligner dans cette section est la suivante : l'affaiblissement considérable de la force incitative lorsqu'on passe d'un équilibre dominant à un équilibre de Nash n'est significative et fructueuse que si les espaces de messages sont plus généraux que les espaces de caractéristiques ${ }^{3}$. En effet, nous avons :

3. Il y a également la possibilité de concrétisation par des équilibres de $\mathrm{Nash}$ de mécanismes directs où les agents mentent. La spécificité de l'espace des caractéristiques comme espaces de messages est alors perdue, puisque le rôle de la vérité disparaît. On peut considérer qu'il s'agit donc même dans ce cas d'un espace de messages abstrait. 


\section{Théorème 2 .}

Une fonction de choix social $f(\cdot)$ est concrétisable en équilibres de Nash par un mécanisme direct révélateur si, et seulement si, elle est concrétisable en équilibres dominants par un mécanisme direct révélateur.

Démonstration. La condition suffisante est évidente par les définitions. Pour la condition nécessire, écrivons que $f($.$) est concrétisée$ par les équilibres de Nash d'un mécanisme direct révélateur ( $g$, (ND)

$$
\begin{array}{r}
\forall i=1 \ldots \mathrm{I} \\
\forall \theta^{i} \in(\mathbb{H})^{i}, \quad \forall \theta^{-i} \in \prod_{j \neq i} \mathrm{H}^{j}
\end{array}
$$

(3)

$$
g\left(\theta^{i}, \theta^{-i}\right) \mathbf{R}^{i}\left(\theta^{i}\right) g\left(\tilde{\theta}^{i}, \theta^{-i}\right), \forall \tilde{\theta}^{i} \in\left(\mathbb{H}^{i}\right.
$$

(3) peut-être réécrit immédiatement :

$$
\begin{gathered}
\forall i=1, \ldots, \mathrm{I} \\
\left.g\left(\theta^{i}, \theta^{-i}\right) \mathrm{R}^{i}\left(\theta^{i}\right) \quad g\left(\tilde{\theta}^{i}, \theta^{-i}\right), \forall \tilde{\theta}^{i} \in(\mathrm{H})^{i}, \forall \theta \in \mathbb{H}\right)
\end{gathered}
$$

c'est-à-dire la définition que $\theta^{i}$ est une stratégie dominante pour l'agent $i$.

Q.E.D.

Dans la section suivante, nous montrons qu'en l'absence de restriction à priori sur les caractéristiques, la concrétisation en équilibres dominants est essentiellement impossible.

\section{CARACTERISATION DES FONCTIONS \\ DE CHOIX SOCIAL CONCRETISABLES EN EQUILIBRES DOMINANTS SOUS LHYPOTHESE DE DOMAINE UNIVERSEL}

Considérons un exemple à trois agents et trois états sociaux $\left\{a_{1}, a_{2}, a_{3}\right\}$ avec le profil de préférences suivant :

$$
\begin{array}{lllll}
a_{1} & \mathrm{P}^{1} & a_{2} & \mathrm{P}^{1} & a_{3} \\
a_{3} & \mathrm{P}^{2} & a_{1} & \mathrm{P}^{2} & a_{2} \\
a_{2} & \mathrm{P}^{3} & a_{3} & \mathrm{P}^{3} & a_{1}
\end{array}
$$


Cet exemple classique permet d'exposer le paradoxe de Condorcet. Si MS désigne la relation binaire, préféré à la majorité simple, on a :

$$
a_{1} \mathrm{MS} a_{2}, a_{2} \mathrm{MS} a_{3}, a_{3} \mathrm{MS} a_{1} .
$$

Cette relation binaire n'est donc pas transitive.

Considérons alors la fonction de choix social définie par le vote à la majorité simple sur le couple $\left(a_{1}, a_{2}\right)$ et le vote à la majorité simple sur l'élu du premier vote et $a_{3}$. Si les agents votent selon leurs vraies préférences, $a_{1}$ l'emporte au premier tour et $a_{3}$ au second. Cependant, lagent 1 peut manipuler la procédure en votant $a_{3}$ au premier tour et aussi au second.

Nous allons généraliser ce résultat en montrant que toute fonction de choix social (FCS) à domaine universel et non dictatoriale est manipulable, c'est-à-dire, dans le langage de la section 1 , n'est pas concrétisable en équilibres dominants. D’après le principe de révélation, il suffit de s'intéresser aux mécanismes directs révélateurs.

Soit $\mathbf{\Sigma}(\mathrm{A})$ l'ensemble des ordres sur $\mathrm{A}^{*}$. Soit $\left(g, \mathbf{\Sigma}(\mathrm{A})^{\mathrm{I}}\right)$ un mécanisme direct révélateur; puisque $g(\cdot)$ doit coïncider avec la fonction de choix social $f($.$) , nous identifions f($.$) et g($.$) .$

Le profil $\mathbf{P}=\left(\mathbf{P}^{1}, \ldots, \mathbf{P}^{\mathrm{I}}\right)$ joue le rôle du vecteur $\theta$ de la section précédente.

Une FCS, $f$, est manipulable à $\mathrm{P}=\left(\mathrm{P}^{1}, \ldots, \mathrm{P}^{\mathrm{I}}\right) \in \Sigma(\mathrm{A})^{\mathrm{I}}$ s'il existe $\mathrm{P}^{\prime i} \in \mathbf{\Sigma}(\mathrm{A})$ tel que :

$$
f\left(\mathrm{P}^{1}, \ldots, \mathrm{P}^{\prime i}, \ldots, \mathrm{P}^{\mathrm{I}}\right) \mathrm{P}^{i} f\left(\mathrm{P}^{1}, \ldots, \mathrm{P}^{i}, \ldots, \mathrm{P}^{\mathrm{I}}\right)
$$

Si lon considère que $\mathrm{P}$ représente les vraies préférences, la manipulabilité signifie que lagent $i$, en annonçant $\mathbf{P}^{\prime i}$, peut s'assurer une issue qu'il préfère.

Une FCS, $f$, est motivante (ou concrétisable en équilibres dominants par un mécanisme révélateur direct) s'il n'existe pas de profil de préférences pour lequel elle est manipulable. En d'autres termes, pour tous les ordres annoncés par les autres, le vrai ordre est une stratégie dominante pour l'agent $i$, pour tout $i$.

$$
\begin{aligned}
& \forall \mathrm{P}=\left(\mathrm{P}^{1}, \ldots, \mathrm{P}^{\mathrm{I}}\right) \in[\Sigma(\mathrm{A})]^{\mathrm{I}}, \forall \mathrm{P}^{i} \in \Sigma(\mathrm{A}) \\
\Rightarrow \quad & f\left(\mathrm{P}^{1}, \ldots, \mathrm{P}^{i}, \ldots, \mathrm{P}^{\mathrm{I}}\right) \mathrm{P}^{\mathrm{i}} f\left(\mathrm{P}^{1}, \ldots, \mathrm{P}^{\prime i}, \ldots, \mathrm{P}^{\mathrm{I}}\right)
\end{aligned}
$$

4. Pour simplifier la démonstration, l'analyse est restreinte aux ordres, c'est-àdire aux préordres asymétriques. 


\section{Revue économique}

Si $f$ est une FCS et a une image $A^{\prime} \subseteq A$, on dira $f$ est une FCS à image $A^{\prime}$.

Une FCS à image $A^{\prime}$ est dictatoriale s'il existe un agent dont l'état social favori dans $A^{\prime}$ est toujours le choix social, c'est-à-dire :

$i \in\{1, \ldots, \mathrm{I}\}$ est un dictateur, si pour tout $\mathrm{P} \in[\mathrm{\Sigma}(\mathrm{A})]^{\mathrm{I}}$, et tout $a \in \mathrm{A}^{\prime}$ tel que $a \neq f(\mathrm{P})$, alors $f(\mathrm{P}) \mathrm{P}^{i} a$.

Théorème 3. (Gibbard [1973], Satterthwaite [1975])

Si $A^{\prime}$ a au moins trois éléments, une fonction de choix social à image $\mathrm{A}^{\prime}$, qui est à domaine universel et motivante, est dictatoriale.

\section{Démonstration 5}

Lemme 1. S’il existe $i \in\{1, \ldots, \mathrm{I}\}$ tel que

$$
\begin{aligned}
& f\left(\mathrm{P}^{1}, \ldots, \mathrm{P}^{i}, \ldots, \mathrm{P}^{\mathrm{I}}\right)=a_{1} \\
& f\left(\mathrm{P}^{1}, \ldots, \mathrm{P}^{\prime i}, \ldots, \mathrm{P}^{\mathrm{I}}\right)=a_{2}, a_{1} \neq a_{2}
\end{aligned}
$$

et si

$$
a_{2} \mathrm{P}^{i} a^{1} \text { ou } a_{1} \mathrm{P}^{\prime i} a_{2} \text {. }
$$

alors :

$$
f \text { est manipulable ̀̀ }\left(\mathrm{P}^{1}, \ldots, \mathrm{P}^{i}, \ldots, \mathrm{P}^{\mathrm{r}}\right)
$$

ou à

$$
\left(\mathbf{P}^{1}, \ldots, \mathbf{P}^{\prime i}, \ldots, \mathrm{P}^{\mathrm{I}}\right)
$$

\section{Démonstration}

$\mathrm{Si}$

$$
\begin{array}{lll}
a_{1} \mathrm{P}^{\prime} & a_{2}, f\left(\mathrm{P}^{1}, \ldots, \mathrm{P}^{i}, \ldots, \mathrm{P}^{\mathrm{I}}\right) \mathrm{P}^{\prime i} f\left(\mathrm{P}^{1}, \ldots, \mathrm{P}^{\prime i}, \ldots, \mathrm{P}^{\mathrm{I}}\right) \\
a_{2} \mathrm{P}^{i} a_{1}, f\left(\mathrm{P}^{1}, \ldots, \mathrm{P}^{\prime i}, \ldots, \mathrm{P}^{\mathrm{I}}\right) \mathrm{P}^{i} f\left(\mathrm{P}^{1}, \ldots, \mathrm{P}^{i}, \ldots, \mathrm{P}^{\mathrm{I}}\right)
\end{array}
$$

Lemme 2. Si $f$ est à image $\mathrm{A}^{\prime} \subseteq \mathrm{A}$ et si $\mathrm{B} \subseteq \mathrm{A}^{\prime}$ et si $\mathrm{P}$ est un profil tel que pour tout couple d'états $a_{1}, a_{2}$, avec :

alors :

$$
a_{1} \in \mathrm{B}, a_{2} \in \mathrm{A} \text { et } a_{2} \notin \mathrm{B}, a_{1} \mathrm{P}^{i} a_{2} \text {, pour tout } i \text {, }
$$

$$
f\left(\mathrm{P}^{\mathrm{x}}, \ldots, \mathrm{P}^{\mathrm{I}}\right) \in \mathrm{B} .
$$

5. Cette démonstration est due à David Schmeidler et Hugo Sonnenschein. 


\section{Démonstration}

Supposons le contraire, soit $f\left(\mathrm{P}^{1}, \ldots, \mathrm{P}^{\mathrm{I}}\right)=a_{2} \notin \mathrm{B}$. Puisque $\mathrm{B} \subset \mathrm{A}^{\prime}$, il existe $\left(\mathrm{P}^{\prime 1}, \ldots, \mathrm{P}^{\prime \mathbf{I}}\right) \in[\mathbf{S}(\mathrm{A})]^{1}$ tel que $f\left(\mathrm{P}^{\prime 1}, \ldots, \mathrm{P}^{\prime \mathbf{x}}\right)=a_{\mathbf{1}} \in \mathrm{B}$.

Considérons alors l'ensemble des états sociaux

$$
\left\{a_{3}^{i}\right\} \begin{aligned}
& i=\mathrm{I} \\
& i=0
\end{aligned}
$$

défni par :

$$
a_{3}^{\prime}=f\left(\mathrm{P}^{\prime 1}, \ldots, \mathrm{P}^{\prime i}, \mathrm{P}^{i+1}, \ldots, \mathrm{P}^{\mathrm{I}}\right)
$$

et soit $j$ le dernier entier, tel que $a_{3}^{i} \in \mathrm{B}$; alors

$$
\begin{aligned}
& f\left(\mathrm{P}^{\prime 1}, \ldots, \mathrm{P}^{\prime j}, \mathrm{P}^{\mathrm{j}+1}, \ldots, \mathrm{P}^{\mathrm{I}}\right)=a_{3}^{j} \in \mathrm{B} . \\
& f\left(\mathrm{P}^{\prime 1}, \ldots, \mathrm{P}^{\prime(j-1)}, \mathrm{P}^{j}, \ldots, \mathrm{PI}^{\mathrm{I}}\right)=a_{3}^{j-1} \notin \mathrm{B} .
\end{aligned}
$$

et

$$
a_{3}^{i} \mathrm{P}^{j} a_{3}^{i-1}
$$

ce qui implique que $f$ est manipulable à :

$$
\left(\mathrm{P}^{\prime 1}, \ldots, \mathrm{P}^{\prime(j-1)}, \mathrm{P}^{j}, \ldots, \mathrm{P}^{\mathrm{I}}\right)
$$

Q.E.D.

L'idée de la démonstration est alors de construire, à partir de la Fonction de choix social $f$, une constitution $\mathscr{F}$ qui satisfasse les hypothèses du théorème d'impossibilité de Arrow ${ }^{6}$; ceci permet de conclure que $\mathscr{F}$ est dictatoriale et, par suite, que $f$ est dictatoriale.

Etant donné un profil $\left(\mathrm{P}^{1}, \ldots, \mathrm{P}^{\mathrm{I}}\right)$ et un couple d'états $\left(a_{1}, a_{2}\right)$, on construit un profil modifié $\left(\tilde{\mathrm{P}}^{1}, \ldots, \tilde{\mathrm{P}}^{\mathrm{r}}\right)$ comme suit : $\tilde{\mathrm{P}}^{i}$ est déduit de $\mathrm{P}^{i}$ en déplaçant $a_{1}$ et $a_{2}$ en tête de liste et en conservant l'ordre $\mathbf{P}^{i}$ sur $\left\{a_{1}, a_{2}\right\}$ et sur $\left\{a / a \in \mathrm{A}, a \neq a_{1}, a \neq a_{2}\right\}$. Soit $\Phi a_{a_{1} a_{2}}\left(\mathrm{P}^{i}\right)$ l'ordre $\tilde{\mathrm{P}}^{i}$ ainsi construit.

Pour le couple $\left(a_{1}, a_{2}\right)$, nous définissons alors :

$$
a_{1} \mathrm{P} \quad a_{2} \text { si } a_{1}=f\left(\tilde{\mathrm{P}}^{1}, \ldots, \tilde{\mathrm{P}} \mathrm{I}\right) .
$$

Ce processus répété pour toute paire d'états sociaux donne une relation binaire $P$ et répété pour tout profil admissible donne une cons-

6. Les hypothèses du théorème d'impossibilité de Arrosv sont : Domaine universel (DU), Principe de Pareto (PP), Indépendance des états non pertinents (IENP). 


\section{Revue économique}

titution potentielle, c'est-à-dire une fonction qui associe à tout profil une relation binaire.

$\mathscr{F}$ satisfait l'hypothèse de domaine universel par définition.

$\mathscr{F}$ satisfait (PP) sur $\mathrm{A}^{\prime}$ par le lemme 2.

$\mathscr{F}$ satisfait (IENP), sinon une démonstration analogue à celle du lemme 2 montrerait que $f$ est manipulable. Il reste à montrer que $\mathrm{P}$ est un ordre. Le fait que $\mathrm{P}$ soit total et asymétrique est évident.

Supposons que $\mathrm{P}$ ne soit pas transitive, alors il existe $\mathrm{P}^{1}, \ldots, \mathrm{P}^{\mathrm{r}}, a_{1}, a_{2}, a_{3}$ avec :

$$
\begin{aligned}
& a_{1}=f\left[\Phi a_{1} a_{2}\left(\mathrm{P}^{1}\right), \ldots, \Phi a_{1} a_{2}\left(\mathrm{P}^{\mathrm{I}}\right)\right] \Leftrightarrow a_{1} \mathrm{P} a_{2} \\
& a_{2}=f\left[\Phi a_{2} a_{3}\left(\mathrm{P}^{1}\right), \ldots, \Phi a_{2} a_{3}\left(\mathrm{P}^{\mathrm{I}}\right)\right] \Leftrightarrow a_{2} \mathrm{P} a_{3} \\
& a_{3}=f\left[\Phi a_{1} a_{3}\left(\mathrm{P}^{1}\right), \ldots, \Phi a_{1} a_{3}\left(\mathrm{P}^{\mathrm{I}}\right)\right] \Leftrightarrow a_{3} \mathrm{P} a_{1}
\end{aligned}
$$

Soit $\left(\mathrm{P}^{\prime 1}, \ldots, \mathrm{P}^{\prime 1}\right)$ le profil défini en plaçant $\left(a_{1}, a_{2}, a_{3}\right)$ en haut de liste et en conservant les ordres $\mathrm{P}^{i}$ sur les ensembles $\left\{a_{1}, a_{2}, a_{3}\right\}$ et $\left\{a / a \in \mathrm{A}, a \neq a_{1}, a \neq a_{2}, a \neq a_{3}\right\}$.

Supposons alors que :

$$
a_{1}=f\left(\mathrm{P}^{\prime \prime}, \ldots, \mathrm{P}^{\prime} \mathrm{x}\right)
$$

Soit $\left(\mathrm{P}^{\prime \prime 1}, \ldots, \mathrm{P}^{\prime \prime x}\right)$ le profil obtemu en déplaçant $a_{2}$ en troisième position sur chacun des ordres $\mathrm{P}^{\prime i}$. Puisque $a_{1} \mathrm{P}^{i} a_{3} \Leftrightarrow a_{1} \mathrm{P}^{\prime \prime i} a_{3}, a_{3} \mathrm{P} a_{1}$. et la propriété (IENP) impliquent :

$$
a_{3}=f\left(\mathrm{P}^{\prime \prime 1}, \ldots, \mathrm{P}^{\prime \prime} \mathrm{I}\right)
$$

Soit $a_{4}^{i}=f\left(\mathrm{P}^{\prime \prime \prime}, \ldots, \mathrm{P}^{\prime \prime}, \mathrm{P}^{\prime i+1}, \ldots, \mathrm{P}^{\prime \mathrm{I}}\right), i=1, \ldots, \mathrm{I}$, et soit $j$ le premier entier tel que $a_{4}^{j} \neq a_{1}$.

Si $a^{i}{ }_{4}=a_{3}$, alors $f$ est manipulable par le lemme 1 ;

Si $a^{i}{ }_{4}=a_{2}$, alors $f$ est manipulable par $j$ à $\left(\mathrm{P}^{\prime \prime 1}, \ldots, \mathrm{P}^{\prime \prime}{ }^{j}, \mathrm{P}^{\prime(j+1)}, \ldots, \mathrm{P}^{\prime \mathbf{I}}\right)$.

Par le théorème d'Arrow, $\mathscr{F}$ est dictatoriale. Le dictateur pour $\mathscr{F}$ est clairement un dictateur pour $f$.

$$
\text { Q.E.D. }
$$

A partir de ce résultat négatif fondamental, on peut rechercher des résultats positifs de plusieurs façons. Comme pour le théorème d'impossibilité d'Arrow, on peut examiner les restrictions sur les préférences qui conduisent à des fonctions de choix social non manipulables.

Il existe d'étroites relations entre les restrictions qui permettent de construire une constitution à la Arrow, et celles qui permettent de construire une fonction de choix social non manipulable (voir Kalai et Muller, [1976]). Nous savons que la procédure de majorité pour 
des préférences «unimodiales» conduit à une fonction de bien-être social à la Arrow. Il est facile de vérifier que le meilleur choix de l'agent médian constitue une fonction de choix social non manipulable. En effet, tout mensonge qui ne modifie pas l'agent médian est sans effet et tout mensonge qui change l'agent médian le change dans le mauvais sens ?.

Dans la section suivante, nous verrons comment la restriction à des fonctions d'utilité adlitivement séparables permet également de construire des fonctions de choix social non manipulables.

Une autre direction consiste à affaiblir la notion de non-manipulabilité en recherchant une concrétisation par des équilibres autres que les équilibres dominants ${ }^{3}$, par exemple, les équilibres de Nash.

Une fonction de choix social $f($.$) est monotone, si :$

$$
\begin{aligned}
& \forall \mathrm{R}, \tilde{\mathrm{R}} \in[\Sigma(\mathrm{A})]^{\mathrm{I}}, \forall a \in \mathrm{A}, a \in f(\mathrm{R}) \\
& \forall i \in \mathrm{I}, \forall b \in \mathrm{A}, a \mathrm{R}^{i} b \Longrightarrow a \tilde{\mathrm{R}}^{i} b \\
& \Longrightarrow a \in f(\tilde{\mathrm{R}})
\end{aligned}
$$

c'est-à-dire si $a$ est choisi pour le profil $R$, et si dans le profil $\tilde{\mathrm{R}} a$ progresse pour tous les agents, $a$ est encore choisi pour le profil $\hat{\mathrm{R}}$.

La fonction de choix social $f($.$) ne comprend pas de pouvoir de$ veto. si, et seulement si, $\forall a \in A, \quad i \in I$, pour un profil $R$ tel que :

$$
\forall i \neq i, \forall b \in A, a \mathrm{R}^{j} b,
$$

alors

$$
a \in f(\mathrm{R}) .
$$

Le théorème fondamental suivant permet de caractériser les fonctions de choix social concrétisables en équilibres de Nash.

Théorème 4. (Maskin $[1977]$ )

Considérons la fonction de choix social de $[\Sigma(-A)]^{\mathrm{I}}$ dans $A$ avec $|\mathrm{A}| \geqslant 3$. Si $f$ est concrétisable en équilibres de Nash, elle est monotone.

7. Hervé Moulin [1980] a montré que les seules fonctions de choix social non manipulables sont dans ce cas les "dictateurs positionnels", c'est-à-dire le meilleur choix du premier, deuxième ou Ième agent, lorsque les états sociaux sont classés de gauche à droite sur une droite. [1979].

8. Voir Green et Laffont [1979], chap. v; Dasgupta, Hammond et Maskin 
De plus, si $f($.$) est monotone et ne comprend pas de pouvoir de veto,$ elle est concrétisable en équilibres de Nash.

La démonstration de ce théorème est constructive et consiste à exhiber un mécanisme pour les espaces de messages $\mathrm{M}^{i}=\prod_{i=1}^{\mathrm{I}} \mathbb{H}^{i}, \forall i$, c'est-à-dire où chaque agent doit annoncer des caractéristiques pour tous les agents.

Ceci met en évidence, de façon extrême, la faiblesse de la notion de concrétisation en équilibres de Nash, car rien n'est dit sur la façon d'arriver à ces équilibres de Nash dans les jeux en information incomplète. Or, dans le mécanisme ci-dessus, à l'équilibre de Nash, l'agent $i$ annonce les caractéristiques de tous les agents de la société.

\title{
UN MECANISME NON MANIPULABLE POUR LA DECISION DE PRODUCTION DE BIENS PUBLICS
}

\begin{abstract}
L'intuition du mécanisme peut être donnée à partir de l'enchère de Vickrey.

Considérons un objet indivisible à vendre à un groupe de I agents. Il est possible d'obtenir de ces agents leurs vraies dispositions à payer pour l'objet de la façon suivante.

Chaque agent doit donner sous pli fermé son offre, sachant que l'objet sera donné à celui qui offre le plus, mais il n'aura à payer que le deuxième plus haut prix. Il est facile de vérifier que la vraie disposition à payer est pour chaque agent une stratégie dominante.

Considérons l'agent 1 , sans perte de généralité, et distinguons deux cas selon que l'annonce de la vérité $v^{1}$ conduit l'agent à avoir l'enchère la plus élevée (premier cas) ou non (second cas). Dans le premier cas, il obtient l'objet et paye, le second plus haut prix, soit $u^{2}$. Il ne peut jamais gagner en proposant une autre offre que $v^{\prime}$. Tant qu'il annonce une offre $w^{1}>w^{2}$, rien n'est changé pour lui et il ne gagne ni ne perd rien. S'il annonce $w^{1}<w^{2}$, il n'a plus lobjet et ne paye rien. Toutefois, il perd l'opportmité d'un gain en utilité de $v^{1}-w^{2}$ s'il avait dit la vérité.

Dans le second cas, tant qu'il annonce $w^{1}$ plus petit que la plus forte enchère $w^{2}$, rien ne change pour lui. S'il annonce $w^{2}>w^{2}$, il a l'objet mais paye $w^{2}$ de sorte qu'il a une perte d'utilité égale à $w^{2}-v^{1}$, positif par hypothèse.
\end{abstract}


Le mécanisme ci-dessous est une adaptation aux biens publics de cette idée. dire :

On suppose que les fonctions d'utilité sont quasi linéaires, c'est-à-

$$
\mathrm{U}^{i}\left(x^{i}, y\right)=x^{i}+v^{i}(y), i=1, \ldots, \mathrm{I}
$$

où $x^{i}$ est la consommation de l'unique bien privé et $y$ la quantité de bien public disponible et l'on s'interroge sur l'opportunité de réaliser un bien public de coût nul.

Soit $\vec{x}$ les ressources globales en bien privé.

Donc,

$$
v=\{0,1\}
$$

et nous adoptons la normalisation :

$$
v^{i}(0)=0, \text { avec } v^{i}(1)=v^{i}, i=1, \ldots, I
$$

Une bonne décision est définie par :

$$
y=1 \Leftrightarrow \sum_{i=1}^{I} v^{i} \geqslant 0
$$

On demande à chaque agent qu'elle est sa disposition à payer pour le bien public, $w^{l}$. Soit $\left(w=\left(w^{1}, \ldots, w^{\mathrm{T}}\right)\right)$.

La décision est prise selon la règle suivante :

$$
y(w)=1 \Leftrightarrow \sum_{i=1}^{I} w^{i} \geqslant 0
$$

en annonçant à l'agent $i$ un transfert en bien privé 9:

$$
\begin{aligned}
t_{i}(w) & =-\left|\sum_{j \neq i} w_{j}\right| \operatorname{si}\left(\sum_{j \neq i} w^{j}\right) \cdot\left(\sum_{i=1}^{x} w^{i}\right)<0 \\
0 & =\operatorname{sinon}
\end{aligned}
$$

Il est facile de vérifier que la vérité $w^{d}=v^{t}$ est une stratégie dominante pour chaque agent.

9. L'agent doit, lorsqu'il change la décision par sa réponse, payer le coût qu'il impose aux autres. C'est aussi le cas dans l'enchère de Vickrey; lorsque l'agent prend l'objet (c'est-à-dire l'enlève aux autres), il paie le coût qu'il impose aux autres (c'est-à-dire le deuxième plus haut prix). 


\section{Revue économique}

Nous calculons ci-après la variation d'utilité $\Delta^{i}$ entre la réponse vraie $v^{i}$ et une autre réponse pour tous les cas possibles :

Premier cas

$$
\sum_{j \neq i} w^{i}>0
$$

a)

$$
\begin{gathered}
\sum_{j \neq i} w^{i}+v^{i} \geqslant 0 \quad \sum_{j \neq i}^{\sum} w^{j}+w^{i} \geqslant 0 \\
\Delta^{i}=v^{i}-v^{i}=0
\end{gathered}
$$

b)

$$
\begin{aligned}
& \sum_{j \neq i} w^{j}+v^{i} \geqslant 0 \quad \sum_{j \neq i} w^{j}+w^{i}<0 \\
& \Delta^{i}=v^{i}-\left(-\sum_{j \neq i} w^{j}\right)=v^{i}+\sum_{j \neq i} w^{i} \geqslant 0
\end{aligned}
$$

c)

$$
\begin{gathered}
\sum_{j \neq i} w^{j}+v^{i}<0 \quad \sum_{j \neq i} w^{j}+w^{i}<0 \\
\Delta^{i}=-\sum_{j \neq i} w^{j}-\left(-\sum_{j \neq i} w^{j}\right)=0
\end{gathered}
$$

d)

$$
\begin{gathered}
\sum_{j \neq i} w^{j}+v^{i}<0 \quad \sum_{j \neq i} w^{j}+w^{i} \geqslant 0 \\
\Delta^{i}=-\sum_{j \neq i} w^{j}-v^{i}=-\left(v^{i}+\sum_{j \neq i} w^{j}\right)>0
\end{gathered}
$$

Second cas

$$
\sum_{j \neq i} w^{j} \leqslant 0
$$

(même type de calculs)

Noter que l'on peut, sans modifier les raisonnements ci-dessus, ajouter à la fonction $t^{i}(w)$ n'importe quelle fonction des réponses des autres $w^{-i}=\left(w^{1}, \ldots, w^{i-1}, w^{i+1}, \ldots, w^{x}\right)$. On obtient ainsi la famille des mécanismes de Groves dont les transferts peuvent être réécrits :

$$
\begin{aligned}
t^{i}(w) & =\sum_{j \neq i} w^{j}+h^{i}\left(w^{-i}\right) & & \text { si } \sum_{i=1}^{\mathrm{I}} w^{i} \geqslant 0 \\
& =h^{i}\left(w^{-i}\right) & & \text { si } \sum_{i=1}^{\mathrm{I}} w^{i}<0
\end{aligned}
$$

où $h^{i}($.$) est une fonction arbitraire de w^{-i}$.

Dans cette classe de mécanismes, il existe des mécanismes réalisables, c'est-à-dire tels que $\sum_{i=1}^{\mathrm{I}} t^{i}(w) \leqslant 0$, qui dégagent un surplus 
de revenus et non un déficit pour le décideur social; par exemple le mécanisme décrit plus haut en (4).

Dans cet exemple, l'ensemble des états sociaux A désigne l'ensemble des consommations de bien privé et une quantité de bien public réalisable

$$
\mathbf{A}=\left\{y,\left(x^{1}, \ldots, x^{\mathrm{I}}\right): y \in\{0,1\}, \sum_{i=1}^{\mathbf{1}} x^{i} \leqslant \bar{x}\right\}
$$

L'application

$$
\left(v^{1}, \ldots, v^{\mathrm{I}}\right) \longrightarrow \begin{gathered}
d \\
\begin{array}{c}
d(v) \\
t^{1}(v) \\
\cdots \\
t^{\mathrm{I}}(v)
\end{array}
\end{gathered}
$$

de $\mathrm{R}^{\mathrm{I}}$ dans $\mathrm{A}$, décrit une fonction de choix social concrétisable en équilibres dominants.

Toutefois, il n'existe pas, en général, de fonctions $h^{i}($.$) , telles que$ $\sum_{i=1}^{\mathbf{I}} t^{i}(w)=0$, c'est-à-dire telle que le budget du décideur social soit juste équilibré. Par conséquent, il n'est pas possible de concrétiser en messages dominants une fonction de choix social Pareto optimale, même sous la restriction très forte de quasi-linéarité. La décentralisation cle linformation est incompatible avec loptimalité de Pareto; le problème du Centre doit être formulé explicitement en termes de second rang comme nous en verrons un exemple dans une section prochaine (p. 25).

Pour progresser vers cette formulation, nous étudions dans la section suivante l'approche différentiable (voir Laffont et Maskin [1980]).

\section{L'APPROCHE DIFFERENTIABLE}

Dans cette section, nous utilisons une méthode constructive pour obtenir des mécanismes à message dominants dans le cas de la révélation des préférences pour les biens publics de la section précédente.

Supposons que le bien public est maintenant divisible et que la fonction cle disposition à payer de l'agent $i$ pour le bien public $y$ est paramétrée par $\theta^{i} \in \mathrm{R}$ et s'écrit $v^{i}\left(y, \theta^{i}\right), v^{i}$ continûment clifférentiable. 


\section{Revue économique}

Le Centre connaît la forme fonctionnelle $v^{i}(\cdot, \cdot)$ pour tout $i$, mais pąs la vraie valeur du paramètre $\theta^{i}$. Il construit un mécanisme direct où $\theta^{i}$, éventuellement différent de $\theta^{i}$, est annoncé par l'agent $i$ et l'état social est défini par une fonction de décision de bien public $y^{*}(\theta)$ et des transferts en bien privé $t^{i}(\hat{\theta}), i=1, \ldots, \mathbf{I}$ où $\widehat{\theta}=\left(\widehat{\theta}^{1}, \ldots, \widehat{\theta}^{\mathrm{r}}\right)$.

Pour qu'il soit dans l'intérêt de l'agent $i$ d'annoncer la vérité, $\hat{\theta}^{i}=\theta^{i}$ doit être une solution du problème de maximisation suivant.

$$
\underset{\tilde{\theta}^{i}}{\operatorname{Max}}\left[v^{i}\left(y^{*}\left(\tilde{\theta}^{i}, \tilde{\theta}^{-i}\right), \theta^{i}\right)+t^{i}\left(\tilde{\theta}^{i}, \tilde{\theta}^{-i}\right)\right]
$$

dont la condition du premier ordre est (sous des hypothèses appropriées de différentiabilité) :

$$
\text { (5) } \frac{\partial v^{i}}{\partial y}\left(y^{*}\left(\tilde{\theta^{i}}, \tilde{\theta}^{-i}\right), \theta^{i}\right) \frac{\partial y^{*}}{\partial \theta^{i}}\left(\tilde{\theta}^{i}, \tilde{\theta}^{-i}\right)+\frac{\partial t^{i}}{\partial \theta^{i}}\left(\tilde{\theta}^{i}, \tilde{\theta}^{-i}\right)=0
$$

Une condition nécessaire pour que $\theta^{i}=\theta^{i}$ soit une solution de (5) pour toute annonce des autres

$$
\tilde{\mathbf{\theta}}^{-i} \in \underset{j \neq i}{\mathrm{II}} \mathrm{H}
$$

est

$$
\frac{\partial v^{i}}{\partial y}\left(y^{*}\left(\theta^{i}, \tilde{\theta}^{-i}\right), \theta^{i}\right) \frac{\partial y^{*}}{\partial \theta^{i}}\left(\theta^{i}, \tilde{\theta}^{-i}\right)+\frac{\partial t^{i}}{\partial \theta^{i}}\left(\theta^{i}, \tilde{\theta}^{-i}\right)=0
$$

Si l'on veut que le mécanisme extraie la vérité quelle que soit la vérité, (6) doit être vrai pour tout $\theta^{i}$; (6) est donc une identité en $\theta$ que nous pouvons réécrire :

$$
\frac{\partial v^{i}}{\partial y}\left(y^{*}(\theta), \theta^{i}\right) \frac{\partial y^{*}}{\partial \theta^{i}}(\theta)+\frac{\partial t^{i}}{\partial \theta^{i}}(\theta) \equiv 0
$$

d'où par intégration

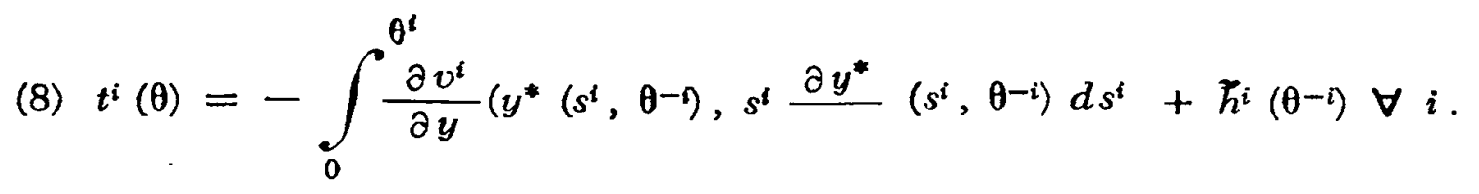

où

$\tilde{h}^{i}\left(\theta^{-i}\right)$ est une fonction arbitraire de $\theta^{-i}$.

Si la fonction de décision de bien public que l'on cherche à concrétiser, $y^{*}(\theta)$, est celle qui maximise $\sum_{i=1}^{\mathrm{I}} v^{i}\left(y, \theta^{i}\right)$ (c'est-à-dire la fonction 
de décision de bien public qui correspond à l'optimum de Pareto intérieur en information parfaite),

$$
\frac{\partial v^{i}}{\partial y}\left(y^{*}(\theta), \theta^{i}\right)=-\sum_{j \neq i} \frac{\partial v^{j}}{\partial y}\left(y^{*}(\theta), \theta^{j}\right)
$$

et (8) devient :

$$
t^{i}(\theta)=\sum_{j \neq i} v^{j}\left(y^{*}(\theta), \theta^{i}\right)+h^{i}\left(\theta^{-i}\right)
$$

c'est-à-dire les mécanismes de Groves dans le cas d'un bien public divisible.

Nous savons que cette forme nécessaire des transferts $t^{i}($.$) conduit$ à des mécanismes pour lesquels la vérité est vraiment une stratégie dominante.

Vérifions-le :

$$
\begin{aligned}
& v^{i}\left(y^{*}\left(\theta^{i}, \theta^{-i}\right), \theta^{i}\right)+\sum_{j \neq 2} v^{j}\left(y^{*}\left(\theta^{i}, \theta^{-i}\right), \theta^{j}\right)+h^{i}\left(\theta^{-i}\right) \geqslant \\
& v^{i}\left(y^{*}\left(\hat{\theta}^{i}, \theta^{-i}\right), \theta^{i}\right)+\sum_{j \neq i} v^{j}\left(y^{*}\left(\hat{\theta}^{i}, \theta^{-i}\right), \theta^{j}\right)+h^{i}\left(\theta^{-i}\right)
\end{aligned}
$$

par définition de $y^{*}($.$) .$

La méthocle exposée ci-dessus est d'une très grande applicabilité dans la théorie des incitations. Elle consiste à intégrer un système différentiel qui exprime des conditions nécessaires pour qu'un mécanisme soit révélateur en stratégies dominantes. En général, des conditions supplémentaires doivent ensuite être imposées aux solutions pour qu'elles satisfassent également aux conditions du second ordre. Dans le cas traité ici, toutes les solutions obtenues par intégration sont valables quand $y^{*}(\cdot)$ maximise $\sum_{i=1}^{\mathrm{I}} v^{i}(\cdot)$.

\section{UNE APPLICATION AUX PRocedures de planification}

Considérons une économie avec I agents, un bien privé et un bien public dont le coût de production est nul, par souci de simplicité.

Les agents ont des ressources initiales en bien privé $w^{i}, i=1, \ldots, I$ et désirent ou non le bien public. 


\section{Revue économique}

Soit une procédure en temps continu qui définit à chaque date $t$ une allocation constituée de consommations de bien privé et d'un choix de bien public

$$
\left(x^{1}(t), \ldots, x^{\mathrm{T}}(t), y(t)\right)
$$

soit

$$
\theta^{i}\left\langle(t)=\frac{\partial U^{i} / \partial y\left(x^{i}(t), y(t)\right)}{\partial U^{i} / \partial x^{i}\left(x^{i}(t), y(t)\right)}\right.
$$

le taux marginal de substitution entre bien public et bien privé de l'agent $i$ à l'instant $t$, où $\mathrm{U}^{i}\left(x^{i}, y\right)$ est la fonction d'utilité de l'agent $i, i=1, \ldots, \mathrm{I}$.

A chaque instant, l'agent $i$ transmet son taux marginal de substitution et la procédure de planification, qui débute à l'instant 0 pour lequel $x^{i}(t)=w^{i}, i=1, \ldots$, I et y $(0)=0$, révise l'allocation selon le système différentiel.

$$
\begin{gathered}
\frac{d y}{d t}(t)=\dot{y}=\mathrm{Y}\left(\theta^{1}(t), \ldots, \theta^{\mathrm{I}}(t)\right) \\
\frac{d x^{i}}{d t}(t)=\dot{x}^{i}=\mathrm{T}^{i}\left(\theta^{1}(t), \ldots, \theta^{\mathrm{I}}(t)\right), \quad i=1, \ldots, \mathrm{I}
\end{gathered}
$$

A chaque instant, l'agent $i$ veut maximiser, par rapport à l'annonce $\hat{\theta}^{i}$, la vitesse d'accroissement de sa fonction d'utilité proportionnèlle à :

$$
\theta^{i} \mathbf{Y}\left(\tilde{\theta}^{i}, \theta^{-i}\right)+T^{i}\left(\tilde{\theta}^{i}, \theta^{-i}\right) 10
$$

Nous dirons que l'agent a un comportement myope.

Une condition nécessaire pour que la vérité soit une stratégie dominante est done :

$$
\theta^{i} \frac{\partial Y}{\partial \theta^{i}}\left(\tilde{\theta}^{i}, \theta^{-i}\right)+\frac{\partial T^{i}}{\partial \theta^{i}}\left(\tilde{\theta}^{i}, \theta^{-i}\right)=0
$$

pour tout $\theta^{i}$ et $\theta^{-i}$.

d'où :

$$
\mathrm{T}^{i}(\theta)=-\int_{0}^{\theta^{i}} s^{i} \frac{\partial \mathrm{Y}}{\partial s^{i}}\left(s^{i}, \theta^{-i}\right) d s^{i}+h^{i}\left(\theta^{-i}\right) .
$$

10. Car

$$
\frac{d U^{i}}{d t}=\frac{a U^{i}}{\partial x^{i}}\left(x^{i}(t), y(t)\right)\left[\theta^{i}(t) \dot{y}+\dot{x}^{i}\right]
$$


La condition nécessaire locale du second ordre est :

$$
\frac{\partial Y}{\partial \theta^{i}}\left(\theta^{i}, \theta^{-i}\right) \geqslant 0, \forall \theta, \forall i .
$$

En fait, cette condition est aussi suffisante car :

$$
\theta^{i} \mathrm{Y}\left(\theta^{i}, \theta^{-i}\right)+\mathrm{T}^{i}\left(\theta^{i}, \theta^{-i}\right) \geqslant \theta^{i} \mathrm{Y}\left(\hat{\theta}^{i}, \theta^{-i}\right)+\mathrm{T}^{i}\left(\hat{\theta}^{i}, \theta^{-i}\right)
$$

pour tout $\left(\hat{\theta}^{i}, \theta^{-i}\right)$.

En effet, en utilisant (11), (12) s'écrit :

$$
\left(\theta^{i}-\hat{\theta}^{i}\right) Y\left(\hat{\theta}^{i}, \theta^{-i}\right) \leqslant \int_{\tilde{\theta}^{i}}^{\theta^{i}} Y\left(s^{i}, \theta^{-i}\right) d s^{i}
$$

qui est vrai en raison de la monotonicité faible de $\mathrm{Y}(\cdot)$.

Cette classe de procédures contient un membre seulement de la classe de procédures MDP ${ }^{11}$ clans le càs où $\mathrm{I}=2$.

Alors :

$$
\begin{aligned}
Y(\theta) & =\theta^{1}+\theta^{2} \\
T^{1}(\theta) & =-\theta^{1} Y(\theta)+\frac{1}{2}[Y(\theta)]^{2} \\
T^{2}(\theta) & =-\theta^{2} Y(\theta)+\frac{1}{2}[Y(\theta)]^{2}
\end{aligned}
$$

qui est la procédure MDP avec partage égal de surplus.

\section{UNE APPLICATION AUX EFFETS EXTERNES}

Considérons l'exemple suivant : une entreprise pollue l'environnement par son activité. Elle peut modifier son niveau de pollution $x$ par une technique de cépollution qui a un coût :

$$
\mathrm{C}(x, \theta)
$$

décroissant en $x$, où $\theta$ représente un paramètre comnu seulement de l'entreprise ; $\theta \in \mathrm{R}$.

11. C'est-à-clire la procéchure proposée par Malinvaucl [1972]. Drèze et de la Vallée l'oussin [1971]. 


\section{Revue économique}

Les dommages créés par un niveau de pollution $x$ sont $\mathrm{D}(x)$.

L'optimum social correspond à un niveau $x^{*}$, tel que :

$$
D\left(x^{*}\right)+C\left(x^{*}, \theta\right)=\min _{x}[D(x)+C(x, \theta)]
$$

Supposons que ce niveau soit unique pour tout $\theta$ et que les fonction $\mathbf{D}($.$) et \mathbf{C}($.$) soient continûment différentiables.$

Quelles sont les fonctions de choix social concrétisables en messages dominants?

Caractérisons un état social par un niveau de pollution $x$ et un transfert $t$ à l'entreprise :

$$
f(\theta)= \begin{cases}x & (\theta) \\ t & (\theta)\end{cases}
$$

En adoptant l'approche différentiable, $(x(\theta), t(\theta))$ est concrétisable si :

$$
\frac{\partial \mathrm{C}}{\partial x}(x(\theta), \theta) \frac{d x}{d \theta}(\theta)+\frac{d t}{d \theta}(\theta) \equiv 0
$$

d'où :

$$
t(\theta)=-\int_{0}^{0} \frac{\partial \mathrm{C}}{\partial x}(x(s), s) \frac{d x}{d s}(s) d s+\text { constante. }
$$

La condition du second ordre local est :

$$
\frac{d x}{d \theta}(\theta) \frac{\partial^{2} \mathrm{C}}{\partial x \partial \theta}(x(\theta), \theta) \geqslant 0, \forall \theta
$$

Supposons que l'on cherche à implémenter le niveau de pollution qui correspond à l'optimum social.

Alors :

$$
\frac{d}{d x} \mathrm{D}(x)+\frac{\partial \mathrm{C}}{\partial x}(x, \theta)=0
$$

définit implicitement $x(\theta)$ et

$$
\begin{aligned}
t(\theta) & =-\int_{0}^{\theta} \frac{d}{d x} \mathrm{D}(x) \frac{d x}{d t}(s) d s+\text { constante } \\
& =\mathrm{D}(x(\theta))+\mathrm{K}
\end{aligned}
$$


La condition locale du second ordre est satisfaite et l'on retrouve alors un cas particulier du mécanisme de Groves.

Si l'on annonce à l'agent qu'il devra payer à une constante près le montant total du dommage créé, il est conduit à annoncer son vrai paramètre de coût.

Il est intéressant d'observer que ce prix «non linéaire» ou cette taxation non pigouvienne permet dans ce cas de résoudre la difficulté liée à la non-convexité de Starrett [1972] tout en obtenant l'information nécessaire.

\section{TARIFICATION D'UN MONOPOLE DONT ON NE CONNAIT PAS LA FONCTION DE COUT}

Considérons un monopole dont la fonction de coût est

$$
\begin{aligned}
& \mathbf{C}(q, \theta)=c_{0}+\theta q, c_{0}>0, \text { si } q>0 \\
& \text { C }(0, \theta)=0
\end{aligned}
$$

où $\theta$ est un paramètre inconnu du centre, $\theta \in[0,1]$.

D’après le principe de révélation, nous savons que toute politique de régulation est identifiable à un mécanisme direct révélateur.

Ignorant les effets revenus, la valeur pour les consommateurs d'une quantité $q$ de bien produit par le monopole est :

$$
V(q)=\int_{0}^{q} v(t) d t
$$

où $P($.$) est la fonction inverse de demande supposée décroissante. Le$ surplus du consommateur est donc : $\mathrm{V}(q)-q \mathrm{P}(q)$.

Un état social est caractérisé par une quantité produite et un transfert forfaitaire au producteur. Si un mécanisme $q(\theta), t(\theta)$ est révélateur, la solution du programme :

$$
\operatorname{Max}_{\tilde{\boldsymbol{\theta}}}\left\{q(\tilde{\boldsymbol{\theta}}) \mathrm{P}(q(\tilde{\boldsymbol{\theta}}))-c_{0}-q(\tilde{\boldsymbol{\theta}}) \boldsymbol{\theta}+\boldsymbol{t}(\tilde{\boldsymbol{\theta}})\right\}
$$

est $\theta$, d'où la condition du premier ordre, transformée en identité

$$
\frac{d q}{d \theta}(\theta) \mathrm{P}(q(\theta))+q(\theta) \frac{d q}{d \theta} \frac{d \mathrm{P}}{d q}-\theta \frac{d q}{d \theta}+\frac{d t}{d \theta} \equiv 0
$$




\section{Revue économique}

et

$$
\begin{aligned}
t(\theta) & =\int_{0}^{0} \frac{d q}{d t}(t)\left[(t)-\mathrm{P}(q(t))-q(t) \frac{d \mathbf{P}}{d q}(t)\right] d t+\hat{\mathrm{K}} \\
& =\theta q(\theta)-q(\theta) \mathrm{P}(q(\theta))-\int_{0}^{\theta} q(t) d t+\tilde{\mathrm{K}}
\end{aligned}
$$

La condition locale du second ordre s'écrit $\frac{d q}{d \theta} \leqslant 0$, et: il est facile de vérifier que, pour un transfert défini par (13), c'est aussi une condition suffisante.

Le profit de lentreprise est alors :

$$
\Pi(\theta)=\mathrm{K}+\int_{\theta}^{1} q(t) d t-c_{0}
$$

et

$$
\frac{d \Pi}{d \theta}=-q(\theta) \leqslant 0
$$

Pour être sûr que l'entreprise ne disparaisse pas, il faut s'assurer que le profit est toujours positif puiscue II ( ) est décroissant en $\theta$; il suffit d'imposer $\Pi(1) \geqslant 0$ ou $\mathrm{K} \geqslant c_{0}$.

Le mécanisme optimal est celui qui maximise la fonction objectif du Centre sous les contraintes incitatives (13), $\frac{d q}{d \theta} \leqslant 0$ et $\Pi \geqslant 0$.

Prenons comme fonction objectil me moyenne pondérée du surplus du consommateur et du profit du monopole et supposons que le Centre a me distribution à priori uniforne sur $\theta$. Il choisit le mécanisme qui maximise l'espérance cle lutilité sociale, c'est-à-dire :

$$
\operatorname{Max} \int_{0}^{1}[V(q(\theta))-p(\theta) P(q(\theta))-t(\theta)] d \theta+\alpha \int_{0}^{t} I I(\theta) d \theta 12
$$

sous les contraintes incitatives.

12. $x$ représente le poids attribuc au profit die monopole. 
En utilisant (13), le programme du Centre peut être réécrit :

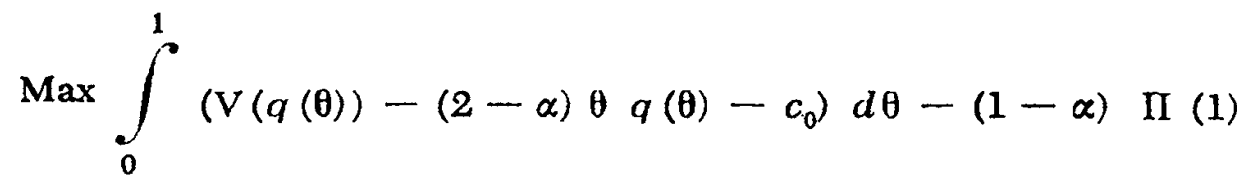

'T. Q. $\quad \Pi(1) \geqslant 0$

$$
q^{\prime}(\theta) \leqslant 0
$$

dont la solution est :

$$
\begin{gathered}
\Pi(1)=0 \\
\frac{d V}{d q}(q)=(2-\alpha) \theta \text { qui donne } q(\theta)=\left(\frac{\partial V}{\partial q}\right)^{-1}((2-\alpha) \theta)
\end{gathered}
$$

qui est bien décroissante.

Le mécanisme obtenu s'interprète comme une règle de tarification au coût marginal modifié.

et un transfert

$$
\tilde{\boldsymbol{p}}(\theta)=c_{m}(\theta)=(2-\alpha) \theta
$$

$$
t(\theta)=\theta q(\theta)-q(\theta) \mathrm{P}(q(\theta))+\int_{\theta}^{1} q(t) d t+c_{0}
$$

qui est à comparer à la règle de tarification optimale en information parfaite composée d'une tarification au coût marginal

$$
p^{*}(\theta)=\theta
$$

et d'un transfert égal au con̂t fixe $c_{0}$ lorsque $\alpha \leqslant 1$.

Le bien-être social obtenu en information imparfaite est inférieur à celui obtenu en information parfaite, car un tarif incitatif pour le monopole conduit à s'écarter de la règle de premier rang prix égale coût marginal (Cf. Baron et Myerson [1979]).

Dans cet exposé, nous avons présenté la concrétisation en équilibres dominants et équilibres de Nash et nous avons illustré par quelques exemples choisis en économie publique la concrétisation en équilibres dominants qui correspond à la solution la plus satisfaisante du point de vue des incitations individuelles. La caractérisation des mécanismes 


\section{Revue économique}

incitatifs est dans ce cas facilement obtenue par l'approche différentiable et la contrainte incitative apparaît alors comme une contrainte de second rang bien définie.

L'immensité du champ de la théorie des incitations provient de la multitude de façon que l'on peut utiliser pour affaiblir les exigences incitatives, par rapport à la concrétisation en équilibres dominants. Si le Centre comnaît les anticipatious de l'agent $i$ concernant les réponses des autres et croit que l'agent $i$ maximise l'espérance d'utilité, il peut se contenter de rechercher une concrétisation en équilibres bayésiens, c'est-à-dire où liannonce de chaque agent est déterminée par la maximisation de son espérance d'utilité. Dans la concrétisation en équilibres maximum, chaque agent maximise son utilité en supposant que les autres agents annoncent ce qui est le plus défarorable pour lui. Une autre direction, utilisée p. 21 , consiste à supposer que les agents sont myopes, c'est-à-dire ne maximisent que le taux instantané d'accroissement de l'utilité.

La notion de fonction de choix social peut être généralisée. On peut étudier des correspondances de choix social et exiger seulement que l'image des messages d'équilibre par le mécanisme appartienne à l'image de cette correspondance. Une autre voie considère des mécanismes aléatoires qui utilisent l'aléa pour améliorer la performance du mécanisme en movenne, ce qui est souvent possible. Je mentionnerai enfin que seul le problème des incitations individuelles a été abordé ici.

JEAN-JACQUES LAFFONT

Professeur à l'Université des Sciences

Sociales de Toulouse

Directeur d'Etudes à l'EHESS 


\section{BIBLIOGRAPHIE}

[1979] Baron D. et Mrensox R., "Regulating a monopolist with unknown costs ", Northwestern U., DP" \# 412.

[1979] Dasgup'a P., Hammovi) P. ('M Mskin E., "The implementation of social choice rules. Some general results on incentive compatibility ", The Review of Economic Studies, 66, p. 185-216.

[1971] Drèze J. et Vallée Poussin D. de la, "A tatonnement process for public goods ", Review of Econonic Studies, 38, p. 133-150.

[1973] Grabard A., "Manipulation for voting schemes ", Econometrica, 41, p. 587 601.

[1979] Green J. et Laffont J.-J., Incentives in public decision making, vol. I, Studies in Public Economies, North Holland.

[1976] Kalar E. et Muller E., "Characterization of domains admitting uon dictatorial social welfare functions and non manipulable voting procedures ", Northwestern U DP.

[1980] Laffont J.-J. et MASkin E., "A differential approach to dominant strategy mechanisms ", Econometrica, 48, p. 1507-1520.

[1981] LafFont J.-J. et Maskin E., "The theory of incentives : on overview ". GREMAR, 8102, Université des Sciences Sociales de Toulouse.

[1972] Malinvaud E., "Prices for individual consumption, quantity indicators for collective consumption ", Review of Economic Studies, 39, p. 385405.

[1977] MASkIN E., "Nash equilibrium and welfare optimality ", Mathematic; for Operations Research, forthcoming.

[1980] Moulin H., "On strategy proofness and single peakedness ", Public Choice, 35, p. 437-455.

[1975] Satrerthwaite M., "Strategy-proofness and Arrow's conditions : existence and correspondence theorems for voting procedures and social welfare functions ", Journal of Economic Theory, 10, p. 187-217.

[1972] Starretr P., "Fundamental non convexities in the theory of externalities", Journal of Economic Theory, 4, p. 180-199. 